\title{
The differentially regulated genes $T V Q R 1$ and TvPirin of the parasitic plant Triphysaria exhibit distinctive natural allelic diversity
}

Quy A Ngo ${ }^{1,2^{*}}$, Huguette Albrecht ${ }^{3 \dagger}$, Takashi Tsuchimatsu ${ }^{4 \dagger}$ and Ueli Grossniklaus ${ }^{2}$

\begin{abstract}
Background: Plant parasitism represents an extraordinary interaction among flowering plants: parasitic plants use a specialized organ, the haustorium, to invade the host vascular system to deprive host plants of water and nutrients. Various compounds present in exudates of host plants trigger haustorium development. The two most effective haustorium inducing factors (HIFs) known for the parasitic plant Triphysaria versicolor (T. versicolor) are peonidin, an antioxidant flavonoid, and 2,6-dimethoxybenzoquinone (DMBQ), an oxidative stress agent. To date, two genes involved in haustorium initiation in T. versicolor have been identified: TVQR1, a quinone oxidoreductase that generates the active HIF from DMBQ, and TvPirin, a transcription co-factor that regulates several other DMBQresponsive and -non-responsive genes. While the expression of these genes in response to DMBQ is well characterized, their expression in response to peonidin is not. In addition, the pattern of polymorphisms in these genes is unknown, even though nucleotide changes in TVQR1 and TvPirin may have contributed to the ability of T. versicolor to develop haustoria. To gain insights into these aspects, we investigated their transcriptional responses to HIFs and non-HIF and their natural nucleotide diversity.

Results: Here we show that TVQR1 and TvPirin are transcriptionally upregulated by both DMBQ and peonidin in $T$. versicolor roots. Yet, while TVQR1 also responded to juglone, a non-HIF quinone with toxicity comparable to that of DMBQ, TvPirin did not. We further demonstrate that TvPirin encodes a protein shorter than the one previously reported. In the T. versicolor natural population of Northern California, TVQR1 exhibited remarkably higher molecular diversity and more recombination events than TvPirin, with the highest non-synonymous substitution rate in the substrate recognition and catalytic domain of the TVQR1 protein.

Conclusion: Our results suggest that TVQR1 and TVPirin have most likely evolved highly distinct roles for haustorium formation. Unlike TvPirin, TVQR1 might have been under diversifying selection to maintain a diverse collection of polymorphisms, which might be related to the recognition of an assortment of HIF and non-HIF quinones as substrates for successful haustorial establishment in a wide range of host plants.
\end{abstract}

Keywords: Parasitic plants, Haustorium, Nucleotide diversity, Allelic polymorphism, Population genetics, Transcriptional responses

\footnotetext{
* Correspondence: qango@access.uzh.ch

${ }^{\dagger}$ Equal contributors

${ }^{1}$ Genetics Graduate Group, University of California - Davis, One Shields

Avenue, Davis, CA 95616, USA

${ }^{2}$ Institute of Plant Biology \& Zürich-Basel Plant Science Center, University of

Zürich, Zollikerstrasse 107, CH-8008, Zürich, Switzerland

Full list of author information is available at the end of the article
} 


\section{Background}

The plant kingdom is usually associated with autotrophy as most plants produce their own nutrients via photosynthesis. Plant parasitism presents a divergence from this generalization as parasitic plants derive all or part of their nutrients and water from their host plants. About 4,000 parasitic plant species are widely distributed among various taxa and over diverse environments, ranging from arctic to tropical climates. Some of the best known parasitic plants include the Christmas ornament mistletoe, the world largest blooming flower Rafflesia, the fragant oil producing sandalwood, and the debilitating agricultural weeds dodder, witchweed (Striga) and broomrape (Orobanche). Although diverse in morphology, reproductive aspects, and life habitats, all parasitic plants share a specialized organ - the haustorium which they evolved to assure a successful life cycle [1-4]. Parasitic plants develop haustoria either near their root tips or along their stems in response to selective chemical factors released by their hosts $[5,6]$. The haustorium initiates via localized cell expansion and division that is coupled with haustorial hair growth in many species. Following attachment and penetration of the host's roots or stems, the haustorium establishes a vascular continuity between the parasite and its host through which water and nutrients are channeled to the parasite to benefit its own growth and development. By so doing, parasitic plants disturb many developmental and physiological aspects of host plants $[1,2,5,6]$. Consequently, parasitic plants have a tremendous impact on community ecology among plants, animals, microbes, and even the surrounding physical environment [7]. Therefore, studies on parasitic plants encompass a range of interests, including anatomy and development, cellular physiology, gene regulation, population genetics, phylogeny, ecology and evolution. Here, we focus on a variety of aspects of host perception and recognition by parasitic plants.

The perception and recognition by parasitic plants of haustorium inducing factors (HIFs) released by host plants is best documented in root parasites of the Orobanchaceae family (many previously classified with Scrophulariaceae). The first three HIFs identified, xenognosin (i.e., host recognition factor) A, xenognosin $\mathrm{B}$, and 2,6-dimethoxybenzoquinone (DMBQ), are phenolic compounds [8-10]. Additionally, various quinone and flavonoid HIFs were subsequently identified and characterized $[11,12]$. Further studies on HIF biogenesis, activity, and recognition in the two root hemiparasites Striga spp. and Triphysaria spp. have shed light onto the chemical signaling by $\mathrm{DMBQ}$, which is involved in the first steps of the haustorium developmental pathway [13-16]. The genus Striga contains prolific weeds causing substantial damage to cereal crops mostly in
Africa [17,18], whereas Triphysaria is a wild native genus in the grassland communities of Northern American coastlands [19]. The current model for haustorium initiation proposes that certain unidentified peroxidases or laccases released by parasitic roots catalyze the production of DMBQ at the host contact site, which diffuses towards the parasitic plant $[14,15]$. Next, a quinone oxidoreductase from the parasite converts DMBQ from its inactive form into the transiently active single-electron free radical form with the suitable redox potential for haustorium induction. This process is hypothesized to be the first step of the haustorium organogenesis pathway $[13,16]$.

To date, only two parasitic plant genes involved in haustorium development have been identified, TvQR1 and TvPirin, both from the root parasite Triphysaria versicolor (T. versicolor) $[13,20]$. The TvPirin protein is a general transcription co-factor that up-regulates several DMBQresponsive and-non-responsive genes [20]. TvQR1 encodes the aforementioned quinone oxidoreductase, which catalyses the reduction of quinones [13]. In comparison to the HIF DMBQ, this enzyme displays even higher catalytic activities for three other non-HIF quinones, one of which is juglone [13]. Consistent with its biochemical role, TvQR1 gene expression is rapidly upregulated in $T$. versicolor root tips exposed to either maize or Arabidopsis root exudates containing active HIFs, DMBQ or the non-HIF juglone $[11,21,22]$. By contrast, up-regulation of TvPirin in root tips only occurs in response to DMBQ and Arabidopsis root exudates [21].

Most identified HIFs are quinones like DMBQ, which are known to be abundant in plant exudates and have also been associated with allelopathic effects (i.e., inhibition of neighboring plant growth and development) and defense against pathogens [23]. At concentrations below $10^{-4} \mathrm{M}$, quinone HIFs induce haustorium development in parasitic plants $[8,11,24,25]$. However, at concentrations between $10^{-4} \mathrm{M}$ and $10^{-3} \mathrm{M}$, they impart the negative effects of oxidative stress to parasitic plants that result in root necrosis and a reduction in the frequency of haustorium formation $[8,11,24,25]$. Further underscoring this quinone toxicity is the enrichment of transcripts involved in oxidative stress and detoxification pathways in T. versicolor root tips following exposure to DMBQ or host root exudates [21]. Like DMBQ, juglone is a wellknown allelopathic compound present copiously in root exudates of the black walnut tree [26], but unlike DMBQ, juglone lacks the haustorium-inducing capability $[21,24]$. The fact that juglone is a better substrate for TvQR1 than DMBQ [13] correlates with the faster and stronger upregulation of $T \nu Q R 1$ expression in $T$. versicolor root tips upon juglone exposure [21,22]. The only non-quinone HIF known to date is the flavonoid peonidin [11]. Peonidin belongs to the anthocyanin class of compounds 
present in red colored flowers and fruits that act as potent antioxidants by scavenging free radicals, and play various roles in plant development and stress protection [27-29]. Although peonidin is a HIF as potent as DMBQ for $T$. versicolor [11], its role in the regulation of $T \nu Q R 1$ and TvPirin gene expression during haustorium development has not yet been investigated. Furthermore, the availability of peonidin, a non-toxic HIF, DMBQ, a toxic HIF, and juglone, a toxic non-HIF, offers the opportunity to utilize these three chemicals to de-couple the two seemingly intertwined pathways triggered by the quinone HIFs namely the oxidative stress response and haustorium organogenesis.

To understand how TvPirin and TVQR1 contribute to the perception of various phytochemicals present in the rhizosphere and, thus, to the initiation of haustorium development, it is essential to survey their patterns of polymorphism in natural populations of $T$. versicolor. Since so far only a single allele of each of these two genes has been reported [13,20-22], we examined the pattern of nucleotide polymorphism of TvQR1 and TvPirin by using $20 T$. versicolor individual plants from natural populations of the Northern Californian grasslands. We found strikingly higher allelic diversity in TvQR1 compared to TvPirin, with the highest nonsynonymous substitution rate in the catalytic domain of the TVQR1 protein. In addition, we provide evidence that TvPirin encodes a protein shorter than the one previously reported [20], and show that TvQR1 and TvPirin are differentially regulated by HIFs and non-HIF. Our results suggest that TvQR1, unlike TvPirin, has maintained a high level of nucleotide diversity, which might reflect its important role in the perception of various phytochemicals from host plants.

\section{Results and discussion}

TVQR1 and TvPirin are differentially regulated by haustorium inducers and non-inducers

To compare the regulation of TVQR1 and TvPirin, we selected two HIFs and a non-HIF displaying overlapping and differential biological properties on parasitic plants: DMBQ with the dual role of an oxidative stress agent and haustorium inducer, peonidin as a non-toxic HIF, and juglone as a non-HIF quinone and strong oxidative stress agent. In $T$. versicolor in vitro haustoria formation assays, both DMBQ and juglone reduced haustorium formation frequency and caused root necrosis at concentrations of $\geq 50 \mu \mathrm{M}$ (Table 1 and Figure 1A), in agreement with previous observations $[11,24]$. Although either $10 \mu \mathrm{M}$ DMBQ or $30 \mu \mathrm{M}$ juglone alone did not result in root necrosis or a reduced frequency of haustorium formation, the combination of both treatments did (Table 1), further substantiating quinone toxicity to the parasite. On the other hand, peonidin had no toxic effects on $T$. versicolor, even at $1 \mathrm{mM}$ concentration (Table 1 and Figure 1A). Using Northern blot hybridizations, we examined the spatial and temporal pattern of transcript levels of TvQR1 and TvPirin in response to DMBQ, peonidin, and juglone in three areas of $T$. versicolor seedlings - root tips, the remainder of the roots, and shoots - at 1, 3, and 5 hours (h) after treatment. TvQR1 was more strongly up-regulated by the non-HIF juglone than by the HIF DMBQ (Figure 1B), corroborating its higher substrate affinity for juglone [13]. On the other hand, TvPirin was similarly upregulated by both the HIFs DMBQ and peonidin, but not by the non-HIF juglone (Figure 1B). These upregulation patterns were observed only in roots but not in shoots (Figure 1B), consistent with a role for TvQR1 and TvPirin in haustorium development only in the root. We further confirmed the differential expression of $T v Q R 1$ and TvPirin in response to these HIFs and nonHIFs in $T$. versicolor root tips at $2-3 \mathrm{~h}$ post-treatment by reverse transcription quantitative polymerase chain reaction (RT-qPCR) (Figure $1 C$ ). Our results suggest that $T v Q R 1$ is associated with both the oxidative stress response and haustorium initiation, while TvPirin appears to be mainly involved in haustorium development. Determining whether TvPirin is exclusively regulated by HIFs and only involved in haustorium initiation would require the analysis of additional non-toxic HIFs, which so far have not been identified.

\section{TvPirin encodes a $\mathbf{2 8 8}$ amino acid long protein}

The TvPirin gene was initially identified as an incomplete EST (Accession BE574904.1) from a suppressive subtractive hybridization ( $\mathrm{SSH}$ ) library enriched for $T$. versicolor root tip transcripts that are up-regulated by DMBQ [22,30]. To determine the sequence of the full length TvPirin transcript, we performed $5^{\prime}$ and $3^{\prime}$ rapid amplification of cDNA ends (RACE) reactions and RTPCR with primers complementary to both ends of the

Table 1 Effects of different HIFs and non-HIFs on haustorium formation and root necrosis in $T$. versicolor

\begin{tabular}{|c|c|c|c|c|c|c|c|}
\hline Treatment & Water & $\begin{array}{l}\text { DMBQ } \\
10 \mu \mathrm{M}\end{array}$ & $\begin{array}{c}\text { DMBQ } \\
100 \mu \mathrm{M}\end{array}$ & $\begin{array}{c}\text { Juglone } \\
50 \mu \mathrm{M}\end{array}$ & $\begin{array}{c}\text { DMBQ10 } \mu \mathrm{M}+\text { juglone } \\
30 \mu \mathrm{M}\end{array}$ & $\begin{array}{l}\text { Peonidin } \\
30 \mu \mathrm{M}\end{array}$ & $\begin{array}{l}\text { Peonidin } \\
1 \mathrm{mM}\end{array}$ \\
\hline$\%$ responsive plants & 0 & 85 & 60 & 0 & 33 & 78 & 90 \\
\hline $\begin{array}{l}\text { Presence of root } \\
\text { necrosis }\end{array}$ & no & no & yes & yes & yes & no & no \\
\hline
\end{tabular}






RACE clones using a pooled RNA population isolated from $T$. versicolor root tips with haustoria induced by $20 \mu \mathrm{m} \mathrm{DMBQ}$. Alignment of the 5, 6, and 4 independently recovered $5^{\prime}$ RACE, $3^{\prime}$ RACE, and full-length cDNA clones, respectively, revealed a TvPirin open reading frame (ORF) encoding a 288 amino acid (aa) long protein with a $5^{\prime}$ untranslated region (UTR) of 64-68 base pairs (bp) (Additional file 1). However, the previously published TvPirin cDNA sequence (Accession JN606867 in [20]; clone TrVeBC2_244713 in [31] and in http://ppgp.huck.psu.edu/search.php) has a coding capacity for a 322 aa long protein [20]. This cDNA sequence was created by contig assemblies following 454 and Illumina sequencing of the $T$. versicolor transcriptome [31]. In comparison to the sequence of the TvPirin full length cDNA reported here (Additional file 2), that of the TrVeBC2_244713 clone contains an additional $80 \mathrm{bp}$ in the $5^{\prime}$ UTR and an extra $100 \mathrm{bp}$ in the $3^{\prime} \mathrm{UTR}$ (http://ppgp.huck.psu.edu/search.php; [31]). The extended $5^{\prime} \mathrm{UTR}$ in clone TrVeBC2_244713 contains an ATG codon that was assigned as the translation start codon and explains the longer coding sequence of the previously published TvPirin ORF (Accession JN606867 in [20]). In order to resolve these discrepancies regarding the TvPirin transcript $5^{\prime} \mathrm{UTR}$ and ORF, we performed in silico experiments and quantitative transcriptional assays.

First, BLAST-searches for the presence of other TvPirin contig assemblies from the $T$. versicolor transcriptome (http://ppgp.huck.psu.edu/search.php, [31]) uncovered seven clones with homology to the TvPirin cDNA clone TrVeBC2_244713 (Additional file 2A). Two of these clones (TrVe62GuB1_70666 and TrVe3GB1_60414) had the same orientation as the TvPirin cDNA and lacked the extended $5^{\prime} \mathrm{UTR}$. The remaining 5 clones had the reverse orientation and $5^{\prime}$ UTRs of variable lengths. One of these clones (TrVeGnuB1_1027) had the same length and shared $100 \%$ identity in reverse complementation order to the TrVeBC2_244713 TvPirin cDNA. Another reversecomplemented clone (TrVe61FuB1_8246) shared discontinuous identity with the TvPirin cDNA sequences in both orientations (Additional files $2 \mathrm{~A}$ and 3). These observations suggest that these seven clones represent either transcripts from genes other than TvPirin or artifacts of the contig assembly. Similar BLAST-searches in the T. versicolor SSH root tip contig libraries enriched for transcripts up-regulated by DMBQ (http://www. plantsciences.ucdavis.edu/yoder/lab/Sequence_index.html) identified two clones, Contig5118 and Contig492, with homologies to the $5^{\prime}$ and $3^{\prime}$ half of our TvPirin cDNA sequence, respectively, and without the extended $5^{\prime} \mathrm{UTR}$ of the TrVeBC2_244713 clone (Additional file 2A).

Second, we performed RT-qPCR on total RNA isolated from $T$. versicolor root tips following exposure to two HIFs (DMBQ and peonidin) and a non-HIF (juglone), 
using primers chosen for amplification of either the extended $5^{\prime} \mathrm{UTR}$ or the shorter $5^{\prime} \mathrm{UTR}$ of the TvPirin transcripts. The shorter $5^{\prime} \mathrm{UTR}$ amplicon displayed the same up-regulated expression pattern as the TvPirin ORF amplicon, whereas the prolonged $5^{\prime}$ UTR amplicon did not show any significant up-regulation in response to either DMBQ or peonidin (Figure 1C), indicating that the extended $5^{\prime}$ UTR does not authentically belong to the TvPirin transcript.

Collectively, our in silico and RT-qPCR results support that the shorter $5^{\prime} \mathrm{UTR}$ is the bona-fide $5^{\prime} \mathrm{UTR}$ of the full-length TvPirin cDNA. Consequently, the TvPirin protein contains 288 aa (Additional file 1B) and not 322 aa as previously reported [20,31] (Additional file 2B). The formerly published TvPirin cDNA sequence likely represents an artifact of 454 and Illumina sequence assembly. The artifactual nature of the TvPirin TrVeBC2_244713 clone is further supported by the fact that BLAST searches for TvQR1 sequences in the T. versicolor transcriptome (http://ppgp.huck.psu.edu/ search.php, [31]) did not yield any TvQR1 complete ORF, suggesting that caution should be applied when using the $T$. versicolor transcriptome assembled by 454 and Illumina sequencing methods to assign gene structures. Our findings reinforce that for T. versicolor, gene annotation for individual gene studies needs a careful and thorough molecular cloning approach coupled with verification by gene expression analysis until a completely and properly annotated genome is available.

\section{TVQR1 and TvPirin exhibit distinctive natural allelic diversity}

The TvQR1 cDNA (Accession AF304461), originally isolated from the same SSH library used to clone the full-length TvPirin cDNA [20,22,30], is a partial cDNA clone containing the complete ORF but lacking the $5^{\prime}$ and 3 'UTRs. We cloned the full-length $T v Q R 1$ cDNA using the same procedures carried out for TvPirin as described above, and recovered 9 independent fulllength TvQR1 cDNA clones representing 9 different $T v Q R 1$ cDNA alleles (Additional file 4). The aforementioned 4 independently recovered full-length TvPirin cDNA clones also corresponded to 4 different TvPirin cDNA alleles (Additional file 1). In order to understand the patterns of nucleotide polymorphisms of these genes, we conducted a molecular population genetic analysis by using natural strains collected from a population in Northern Californian grasslands (Figure 2). We randomly selected 20 plants from the in vitro haustorium formation assays, of which 6 were responsive to DMBQ (designated as R1-R6), 8 responsive to peonidin ( $r 1-r 8)$, 3 non-responsive to DMBQ (N1-N3), and 3 nonresponsive to peonidin (n1-n3) (see Methods). Genomic sequences from the start codon to the stop codon were

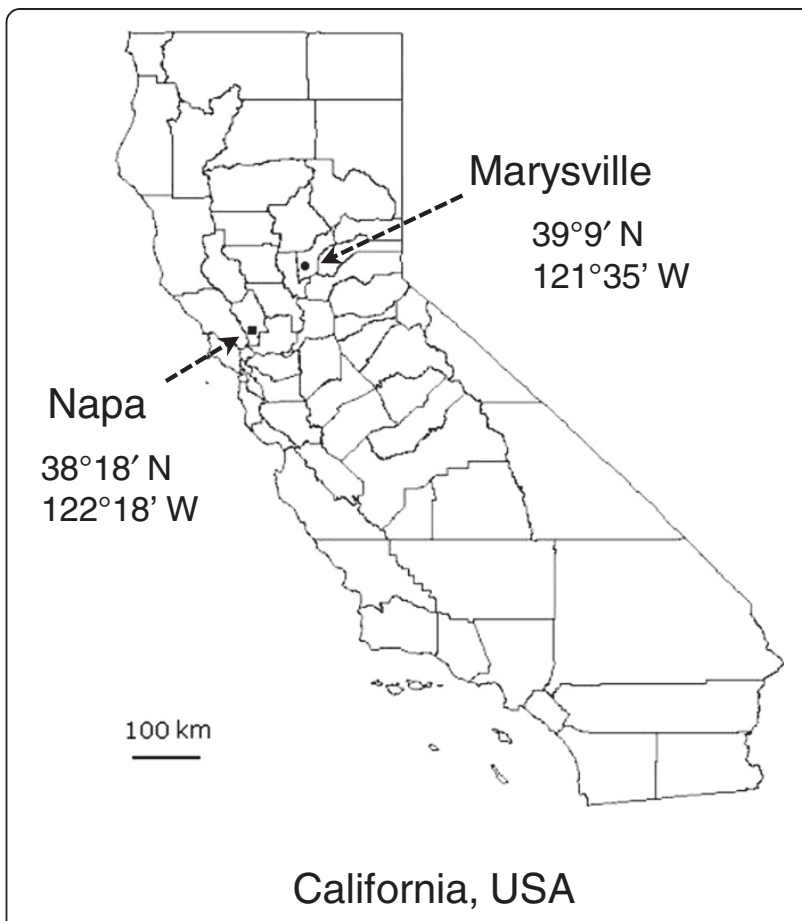

Figure 2 Map showing the $T$. versicolor seed collection sites.

obtained for each gene from the same plant by the Sanger sequencing method.

The complete alignments of all 40 alleles from these 20 plants showed sequences of 1962 and 2011 nucleotides long for TvQR1 and TvPirin, respectively (Additional files 5 and 6). We found striking differences in nucleotide diversity between the two genes. While there were only 9 single nucleotide polymorphisms (SNPs) in TvPirin (Additional file 6, Table 2), TvQR1 contained 350 segregating sites in addition to 31 insertions/deletions (indels) throughout the entire gene body, with indels present only in introns, and with the highest diversity in intron 3 (Additional file 5, Table 2, Figure $3 \mathrm{~A})$. This confers $T v Q R 1$ nucleotide diversity two orders of magnitude higher than that of TvPirin. Likewise, the numbers of synonymous substitutions and non-synonymous changes in TvQR1 are 55 and 27 times higher, respectively, than those of TvPirin (Table 2). Furthermore, four-gamete tests detected a higher level of recombination in TvQR1 $(\mathrm{Rm}=32)$ compared to TvPirin $(\mathrm{Rm}=2) \quad($ Table 2, Figure $3 \mathrm{~B})$, which might have contributed to the generation of the extremely high number of haplotypes in $T v Q R 1$. In $T v Q R 1$, the highest number of recombination events was estimated in the last exon (Figure 3B). As all these individual plants came from one single population of a restricted geographical location (Figure 2), this high level of intra-populational variations of $T v Q R 1$ is even more extraordinary. 
Table 2 Population genetic summary statistics of TVQR1 and TvPirin

\begin{tabular}{|c|c|c|}
\hline & TVQR1 & TvPirin \\
\hline Number of nucleotide sites & 1962 & 2011 \\
\hline Number of segregating sites (S) & 350 & 9 \\
\hline Average number of pairwise differences $(\pi)^{*}$ & 0.09306 & 0.00096 \\
\hline Watterson's estimator of population mutation rate $\left(\theta_{W}\right)$ & 0.04966 & 0.00105 \\
\hline Minimum number of recombination (Rm) & 32 & 2 \\
\hline Number of synonymous sites & 239.02 & 198.19 \\
\hline Number of nonsynonymous sites & 747.98 & 665.81 \\
\hline Number of synonymous and non-coding positions & 909.02 & 1345.19 \\
\hline$\pi_{\mathrm{s}}$ (nucleotide diversity of synonymous sites)* $^{*}$ & 0.12568 & 0.00228 \\
\hline$\pi_{a}$ (nucleotide diversity of nonsynonymous sites)* & 0.01167 & 0.00043 \\
\hline
\end{tabular}

*Jukes-Cantor corrected.

Next, we examined the diversity of $T v Q R 1$ and TvPirin genes at the protein level by aligning the aa sequences deduced from the ORFs of their genomic and full-length cDNA clones. InterProScan runs from Swissprot for these two proteins identified the alcohol dehydrogenase $(\mathrm{ADH})$ GrosE-like and the ADH_N_2 Rossmann domains in TvQR1 (Additional file 7), and the Pirin $\mathrm{N}$ - and C-terminal domains in TvPirin (Additional file 8). Although the crystallized structure of the human Pirin homolog revealed the $\beta$-barrel structure of the $\mathrm{N}-$ and C-terminal domains [32], no biochemical functions for either domain have been identified. By contrast, in redox enzymes such as $\mathrm{ADH}$ or quinone oxidoreductases, the ADH GrosE-like domain is the catalytic site with the oxidoreductase activity and substrate specificity [33,34], while the ADH_N_2 Rossmann domain is the $\operatorname{NAD}(\mathrm{P})$-binding site where the oneelectron addition/reduction reaction takes place [35-37].

As predicted from the higher number of nonsynonymous substitutions described above, the TvQR1 protein exhibited more aa variations than the TvPirin protein (Additional files 7 and 8). A total of 13 aa changes were present in the 288-aa TvPirin protein with no preferential domain of high polymorphic level, and 12 of these variations belonged to rare alleles where the polymorphism occurred only once as singletons in the 34 deduced proteins (Additional file 8). On the other hand, only 13 of the 33 aa variation sites in the 329-aa TvQR1 protein sequence were singletons among the 29 deduced proteins (Additional file 7). The ADH GrosElike domain of TvQR1 presented the largest cluster of aa polymorphisms. Its 14 changes within the 64-aa stretch (22\%) are 3-fold higher than the 17 changes distributed over the remaining 266 aa residues of the protein (6.4\%). Notably, the other domain of TvQR1, ADH_N_2 Rossmann, contained only 9 changes in the 130-aa stretch (7\%), with 2 residues at positions 289 and 305 having 4 and 3 variants, respectively (Additional file 7).
We further looked for aa changes in these two proteins that occurred exclusively in the non-responsive plants in order to identify potential mutations associated with the loss of haustorium formation phenotype. However, such changes were not found (Additional files 7 and 8), suggesting that loss-of-function mutations could reside in the promoter and/or other regulatory elements of the genes.

Collectively, these population genetic analyses revealed a remarkably higher level of molecular diversity, at both nucleotide and aa levels, of TvQR1 compared to TvPirin in the T. versicolor natural populations from Northern California. It is noteworthy that the highest aa polymorphism in TvQR1 appears in the ADH GrosE-like domain, which determines substrate specificity. Thus, by analogy to the extremely divergent LRR domain of the plant resistance $R$ genes involved in specific pathogen recognition [38-43], the ADH GrosE-like domain of TvQR1 may have been under diversifying selection that sustains its molecular labile state in order to enable rapid changes aimed at recognizing a large variety of quinone substrates from host root exudates. At least 19 species belonging to 14 different families in both monocot and dicot clades are natural hosts of $T$. versicolor, and multiple hosts are often parasitized simultaneously by $T$. versicolor in the fields [19]. Therefore, having the ability to recognize a multitude of host factors from such a wide range of hosts would confer this parasitic species an evolutionary advantage. We hypothesize that parasitic plants might have evolved a mechanism to exploit the abundant allelopathic chemicals in their environment and turn the phytotoxicity they initially encountered into a haustorium-inducing cue for their benefit. Maintenance of $T v Q R 1$ molecular diversity could be a mechanism enabling this evolutionary process. High level of nucleotide diversity in synonymous sites as well as in non-synonymous sites of $T \nu Q R 1$ may suggest that the long-term selection has been acting on this gene, as it is 



B

TVQR1

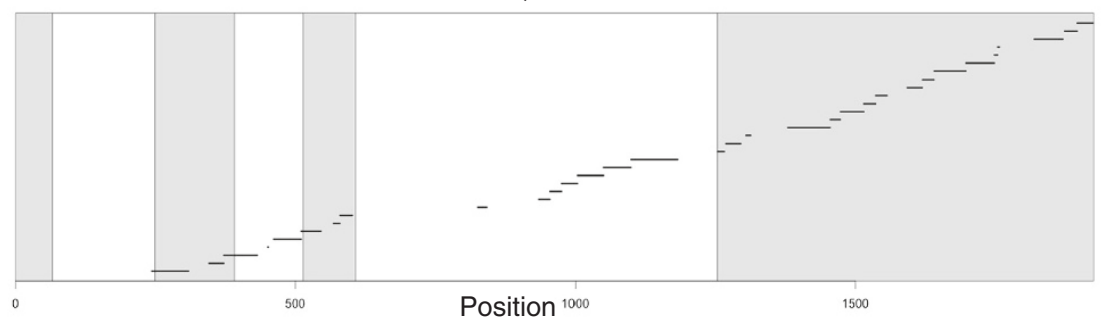

TVPirin

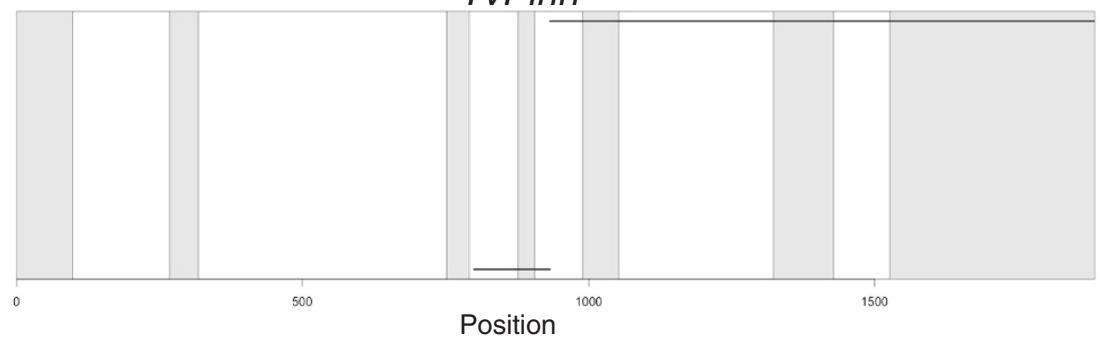

Figure 3 Distinctive natural allelic polymorphisms of TVQR1 and TvPirin. (A) Sliding window analysis of nucleotide diversity of TVQR1 and TvPirin. Solid and dashed lines are silent (synonymous and intron) and non-synonymous nucleotide diversity, respectively. Shaded regions indicate exons. (B) Minimum estimates of recombination across TVQR1 and TvPirin. Within each line, at least one recombination event was detected by the four-gamete test. Shaded regions indicate exons.

expected to elevate nucleotide diversity of closely linked sites while non-synonymous sites are more likely to be the direct target of selection. Those patterns are indeed observed in the plant $\mathrm{R}$ genes that are suggested to be under long-term balancing selection (e.g. [41,42]). TvPirin, on the other hand, as a transcription co-factor, probably interacts with other proteins with specific conformations for DNA binding, and thus would be subject to more evolutionary constraints in order to maintain specific protein-protein interactions, which might have led to the much lower molecular diversity observed here.

\section{Conclusions}

Our molecular analysis has shown that, in the natural T. versicolor populations from Northern California, the 
only two genes known to date to be required for haustorium initiation, $T v Q R 1$ and TvPirin, display highly contrasting patterns of molecular polymorphisms. Especially, the nucleotide diversity of TVQR1 is 97-times higher than that of TvPirin, suggesting that these two genes would have been subjected to different selective pressures, possibly reflecting their distinct roles in phytochemical perception during host recognition. This difference is further manifest in their differential regulation by HIFs and non-HIFs. Since TvQR1 responds to both HIFs and non-HIFs, it might not only function in haustorium development but also in the oxidative stress response. The non-toxic HIF peonidin provides a useful tool to dissect these two pathways in future search for additional parasitic plant genes involved in haustorium development. Differential responses of parasitic genes under peonidin, other toxic HIFs, and toxic non-HIFs bear the potential to separate genes only involved in the detoxification pathway from genes specific to the haustorium signaling pathway. Furthermore, although peonidin is a very effective HIF in T. versicolor, its ability to induce haustoria in other parasitic plant species has not yet been investigated. How peonidin functions as a HIF, e.g., with regard to its active form and receptor on the parasite, also awaits further studies. Both $T v Q R 1$ and TvPirin genes have orthologs in non-parasitic plants. Thus, further phylogenic investigations of these genes in different plant taxa might elucidate the mechanism(s) by which parasitic plants have, during the evolutionary course from complete autotrophy to heterotrophy, co-opted a quinone oxidoreductase and a transcription co-factor from their existing genetic reservoir and adapted them to fulfill the new function of developing the organ that embodies plant parasitism: the haustorium.

\section{Methods}

\section{Plant and chemical materials}

T. versicolor seeds were collected and pooled from wild populations growing in the grasslands of Northern California, USA. For T. versicolor cDNA clones and Northern blot analysis, seeds were collected in Napa (Napa county) (Figure 2). For in vitro haustorium and root necrosis assays, qRT-PCR experiments, and population genetics study, seeds were collected in Marysville (Yuba county) (Figure 2). DMBQ and juglone were obtained from Pflatz \& Bauer (Waterbury, CT, USA) and Alfa Aesar (Karlsruhe, Germany). Peonidin was obtained from Indofine Chemicals (Belle Mead, NJ, USA) and Santa Cruz Biotechnology, Inc. (Heidelberg, Germany).

\section{Haustorium induction and toxicity assays}

T. versicolor seeds were surface-sterilized, germinated, grown on agar plates, and seedlings treated with various haustorial inducers (10 or $100 \mu \mathrm{M}$ DMBQ, $30 \mu \mathrm{M}$ or $1 \mathrm{mM}$ peonidin) and non-inducers (50 $\mu \mathrm{M}$ juglone), treatment combinations $(10 \mu \mathrm{M} \quad \mathrm{DMBQ}+30 \quad \mu \mathrm{M}$ juglone), or mock-treated with water or $0.1 \% \mathrm{EtOH}$ (which is the peonidin solvent) as described previously $[11,21]$. Each plate contained 10-20 seedlings. For each treatment, haustorium formation and root necrosis were scored $24 \mathrm{~h}$ after treatments in 60-66 seedlings.

\section{Nucleic acid extraction}

For Northern blot analysis, total RNA was isolated from root tips, remaining roots, and shoots of 100-200 T. versicolor plants treated with water, $0.1 \%$ ethanol, $20 \mu \mathrm{M}$ $\mathrm{DMBQ}, 10 \mu \mathrm{M}$ juglone, or $30 \mu \mathrm{M}$ peonidin at 1,3 , and $5 \mathrm{~h}$ after treatment. For RT-qPCR, $20 \mu \mathrm{M}$ DMBQ or 30 $\mu \mathrm{M}$ peonidin was applied twice to the same seedling plates at 2-day intervals and haustorium formation was scored $24 \mathrm{~h}$ after each time to identify responsive and non-responsive plants. Then the third treatment was applied, and $2 \mathrm{~h}$ later, root tips were collected from three batches of 20 each inducer-responsive plants representing three biological replicates. One application was performed for mock (water and $0.1 \%$ ethanol) or $10 \mu \mathrm{M}$ juglone treatments to collect root tips for three biological replicates. Total RNA was extracted from root tips by the Trizol method (Invitrogen, Carlsbad, CA, USA). From the haustorium formation assays performed for the RT-qPCR experiments, 20 individual plants responsive or non-responsive to $\mathrm{DMBQ}$ or peonidin were randomly selected for genomic DNA extraction using CTAB and chloroform, followed by isopropanol precipitation and ethanol washes [44].

\section{Transcription assays}

Northern blot hybridization was performed with $10 \mu \mathrm{g}$ of total RNA from each sample as described elsewhere [22]. cDNA probes for the RNA blot were synthesized by PCR-amplifying the TvQR1, TvPirin, and $T v R u v B$ $D N A$ helicase partial cDNA clones from the SSH library [21], randomly labeled by nick-translation with CTP ${ }^{32}$ as per the manufacturer's protocol (Ambion, now Invitrogen, Carlsbad, CA, USA), and used at $10^{7} \mathrm{cpm} / \mathrm{ml}$ of hybridization buffer. The radioactive signals on the filters were captured on the Phosphor screen and images were scanned by the Storm PhosphorImager (GMI, Ramsey, MN, USA). The same filters were sequentially hybridized to different probes following the stripping of each previous probe and exposure to the Phosphor screen to check for residual signals. For RT-qPCR, two technical replicates were carried out with $1 \mu$ of 1:20 diluted cDNAs reverse-transcribed from $2 \mu \mathrm{g}$ each of three total RNA biological replicates in a $20 \mu \mathrm{l}$ volume using SYBR Green I dye in an Applied Biosystem 7500 real-time PCR machine (Applied Biosystem). PCR 
parameters were as followed: $50^{\circ} \mathrm{C} / 20 \mathrm{sec}, 95^{\circ} \mathrm{C} / 10 \mathrm{~min}$, $40 \times\left(95^{\circ} \mathrm{C} / 15 \mathrm{sec}, 60^{\circ} \mathrm{C} / 1 \mathrm{~min}\right)$. Melting curve analysis was done at the end of the PCR reactions to confirm single $\mathrm{PCR}$ product amplifications at $95^{\circ} \mathrm{C} / 1 \mathrm{~min}, 60^{\circ} \mathrm{C} /$ $30 \mathrm{sec}, 95^{\circ} \mathrm{C} / 1 \mathrm{~min}, 60^{\circ} \mathrm{C} / 15 \mathrm{sec}$. Relative quantification of target transcripts were normalized to the reference transcript TvRuvB DNA helicase, which is constitutively expressed, and the relative expression levels of each transcript species under inducing and non-inducing conditions were compared to the control (water) treatment by the $\Delta \Delta \mathrm{Ct}$ method [45].

\section{Gene cloning}

$5^{\prime}$ and $3^{\prime}$ RACE reactions of TvQR1 and TvPirin were performed with total RNA isolated from DMBQresponsive T. versicolor root tips using the Invitrogen's RLM RACE kit per the company protocol. The amplified cDNAs were cloned into the pCR2.1TOPO vector (Invitrogen) and sequenced; the transcriptional start and stop sites of these sequences were identified and used to design new primers complementary to both ends of each cDNA clone for subsequent PCR amplification of the full-length cDNA clones from the same cDNA source used for RACE and from T. versicolor genomic DNA with high-fidelity polymerases $P f u$ or Phusion Taq (NEB). PCR parameters were typically as follows: $94^{\circ} \mathrm{C} /$ $2 \mathrm{~min}, 30 \times\left(94^{\circ} \mathrm{C} / 15 \mathrm{sec}, 60^{\circ} \mathrm{C} / 25 \mathrm{sec}, 72^{\circ} \mathrm{C} / 2.5 \mathrm{~min}\right)$, $72^{\circ} \mathrm{C} / 5 \mathrm{~min}$, with the $94^{\circ} \mathrm{C}$ steps being replaced with $98^{\circ} \mathrm{C}$ when Phusion Taq was used. The full-length cDNA PCR products were cloned into pCRII vector (Invitrogen) and sequenced. The genomic DNA PCR product from each individual plant was directly sequenced by the Sanger sequencing method and the sequence chromatograms visually inspected for sites where two nucleotide peaks of similar height were present which indicated heterozygosity. Sequences of both strands of heterozygotes were visually inspected to confirm the same two nucleotide peaks. The genomic DNA of the identified heterozygotes was subcloned into the pDrive vector, and the corresponding inserts isolated from 2-4 independent clones were subsequently sequenced to identify the SNPs in each allele.

\section{Sequence analyses}

Contig assembly was done manually or by using the CLC Main Workbench 6.6.1 software (CLC bio) coupled with visual inspection for base call error and heterozygo sity detection. BLASTN 2.2.13 and BLASTN 2.2.26+ [46] were used to search for TvPirin sequences in the $T$. versicolor genome sequence databases at http://ppgp.huck. psu.edu/search.php and http://www.plantsciences.ucdavis. edu/yoder/lab/Sequence_index.html. Multiple sequence alignments were performed with MultAlin (http://multalin. toulouse.inra.fr/multalin/ [47] or ClustalW2 (http://www.
ebi.ac.uk/Tools/msa/clustalw2/, $[48,49]$ ), and the multiple alignments presented in the BOXSHADE format (http://www.ch.embnet.org/software/BOX_form.html, Kay Hofmann and Michael D. Baron). The protein PFAM domains were identified from Swissprot database using InterProScan (http://www.ebi.ac.uk/Tools/pfa/iprscan/).

\section{Population genetic analysis}

Sequence alignments were performed using ClustalX installed in MEGA5 [50]. Minor adjustments to optimize the alignments were made by eye. Various population genetic analyses were performed using DnaSP v5 [51]. Nucleotide diversity was corrected using the JukesCantor method [52]. In the sliding window analysis, window length was $50 \mathrm{bp}$ and step size was $10 \mathrm{bp}$.

\section{Primers $\left(5^{\prime}\right.$ to $\left.3^{\prime}\right)$}

$T v Q R 1$ full-length cDNA: forward CTGGAATT

CATTTTCAAGCTTCTCA, reverse CTTATGGCT CGACAACGATTTT.

TvPirin full-length cDNA: forward ATTCCTCAT AAACATCAAATCCCCA, reverse TAGGTTAACAAAA GATCTCATATCAAAACA.

TvQR1 RT-qPCR (164 nt amplicon): forward TGAT AAAGTTGTTGCTATGCTTGGC, reverse GTTGGGT GAGGGCCATGTG.

TvPirin RT-qPCR (90 nt amplicon): forward TCCAAG GACAAAATGATTGAACCT, reverse CACTTTTAC TTCAACCCCATCTTTTT.

Bona-fide TvPirin 5'UTR RT-qPCR (60 nt amplicon): forward ATTCCTCATAAACATCAAATCCCCAAT, reverse ATGATATAACGTCGAGAAGACGGGTTT.

TrVeBC2_244713 5'UTR RT-qPCR (102 nt amplicon): forward GAAATGCCACTATTACTCAGCTGGT, reverse TGGGGATTTGATGTTTATGAGGA.

TvRuvB DNA helicase RT-qPCR (133 nt amplicon): forward TAAACCGAGCTCTGGAGAACGAC, reverse ATGAGCAGACGGTCGAGAAAATC.

\section{Accession numbers}

Sequence data from this article can be found in the GenBank data libraries under accession numbers XX000000.

\section{Additional files}

Additional file 1: Identification of TvPirin full-length CDNA and open reading frame.

Additional file 2: TvPirin open reading frame encodes a 288-amino acid long protein.

Additional file 3: Discontinuous identity between Accession JN606867 and clone TrVe61FuB1_8246.

Additional file 4: Multiple alignment of full-length TVQR1 cDNA clones. 


\section{Additional file 5: Multiple sequence alignment of all genomic TVQR1 alleles.}

Additional file 6: Multiple sequence alignment of all genomic TvPirin alleles.

Additional file 7: TvQR1 protein domains and amino acid diversity. Additional file 8: TvPirin protein domains and amino acid diversity.

\section{Abbreviations}

T. versicolor: Triphysaria versicolor; HIF: Haustorium inducing factor; DMBQ: 2,6-dimethoxybenzoquinone; SNP: Single nucleotide polymorphism; Indel: Insertion/deletion; RT-qPCR: Reverse transcription - quantitative polymerase chain reaction.

\section{Competing interests}

The authors declare that they have no competing interests.

\section{Authors' contributions}

QAN conceptualized and designed the study. QAN, HA, and TT carried out experiments, analyzed the data, and wrote the manuscript. UG provided the infrastructure, research materials and reagents, and analysis tools. All authors critically read and commented on the manuscript and approved the final version for submission.

\section{Authors' information}

QAN studied Triphysaria versicolor and Triphysaria pussilla during her PhD program at the Genetics Graduate Group of the University of California - Davis during the years of 2000-2003, focusing on cloning and functional characterization of Triphysaria candidate genes involved in haustorium development, and on the development of Triphysaria transformation systems for gene functional test by RNAi-mediated gene silencing. Since 2007, QAN is a postdoctoral fellow at the University of Zürich. HA established root exudates as haustorium inducers in $T$. versicolor and identified peonidin as a HIF in T. versicolor during her postdoctoral work from 1993 to 1997 at the University of California Davis. $\Pi$ studies the genetic basis of adaptation from 2010-2011 as a postdoctoral fellow at the University of Zürich and, since 2012, at the Gregor Mendel Institute in Vienna. In this study, he contributed to the analysis of the nucleotide polymorphism in T. versicolor. UG is heading the Department of Plant Developmental Genetics at the Institute of Plant Biology of the University of Zürich and studies developmental, ecological, and evolutionary aspects of plant reproduction.

\section{Acknowledgements}

We thank Dr. John Yoder (University of California - Davis) for providing laboratory space for the Northern blot experiments and gene cloning, Dr. Denneal Jamison, Dr. Jean-Michel Petit, Dr. Russel Wrobel (all at University of California - Davis), and Dr. Philipp Schlüter (University of Zürich) for helpful discussions, the Division of Biological Sciences Sequencing Center (University of California - Davis) and Daniela Guthörl and Dr. Edith Schlagenhauf (University of Zürich) for sequencing assistance, Arturo Bolanos and Peter Kopf (University of Zürich) for general laboratory support. This work was supported in part by a Jastro-Shields graduate grant from the University of California - Davis to QAN. QAN was further supported through teaching assistantships from the Genetics Graduate Group and the Molecular and Cell Biology Department of the University of California - Davis, and the University of Zürich. TT was supported by an EMBO long-term postdoctoral fellowship.

\section{Author details}

${ }^{1}$ Genetics Graduate Group, University of California - Davis, One Shields Avenue, Davis, CA 95616, USA. ${ }^{2}$ Institute of Plant Biology \& Zürich-Basel Plant Science Center, University of Zürich, Zollikerstrasse 107, CH-8008, Zürich, Switzerland. ${ }^{3}$ Department of Public Health Sciences, School of Medicine, University of California - Davis, One Shields Avenue, Davis, CA 95616, USA. ${ }^{4}$ Gregor Mendel Institute, Austrian Academy of Sciences, Dr. Bohr-Gasse 3, 1030, Vienna, Austria.
Received: 29 October 2012 Accepted: 11 February 2013

Published: 18 February 2013

\section{References}

1. Heide-Jorgensen HS: Parasitic Flowering Plants. Leiden, The Netherlands: Brill Academic Publishers; 2008.

2. Kuijt J: The Biology of Parasitic Flowering Plants. Berkeley: University of California Press; 1969.

3. Musselman DL, Press MC: Introduction to parasitic plants. In Parasitic Plants. Edited by Press MC, Graves JD. London: Capman and Hall; 1995:1-13.

4. Nickrent DL, Musselman DL: Parasitic flowering plants. American Phytopathological Society APSnet Education Center, the Plant Health Instructor web publication The Plant Health Instructor; 2004. doi:10.1094/ PHI-I-2004-0330-01.

5. Musselman LJ, Dickison WC: The structure and development of the haustorium in parasitic Scrophulariaceae. Bot J Linnean Soc 1975, 70:183-212.

6. Riopel JL, Timko MP: Haustorial initiation and differentiation. In Parasitic Plants. Edited by Press MC, Graves JD. London: Chapman and Hall; 1995:39-79.

7. Press MC, Phoenix GK: Impacts of parasitic plants on natural communities. New Phytol 2005, 166(3):737-751.

8. Chang M, Lynn DG: The haustorium and the chemistry of host recognition in parasitic angiosperms. J Chem Ecol 1986, 12:561-579.

9. Lynn DG, Steffens JC, Kamut VS, Graden DW, Shabanowitz J, Riopel JL: Isolation and characterization of the first host recognition substance for parasitic angiosperms. J Am Chem Soc 1981, 103:1868-1870.

10. Steffens JC, Lynn DG, Kamat VS, Riopel JL: Molecular specificity of haustorial induction in Agalinis purpurea (L.) Raf. (Scrophulariaceae). Ann Bot 1982, 50:1-7.

11. Albrecht $\mathrm{H}$, Yoder Jl, Phillips DA: Flavonoids promote haustoria formation in the root parasite Triphysaria versicolor. Plant Physiol 1999, 119(2):585-592.

12. Riopel JL, Timko MP: Signals and regulation in the development of Striga and other parasitic angiosperms. Boca Raton: CRC Press; 1992.

13. Bandaranayake PC, Filappova T, Tomilov A, Tomilova NB, Jamison-McClung $D, N g o$ Q, Inoue K, Yoder Jl: A single-electron reducing quinone oxidoreductase is necessary to induce haustorium development in the root parasitic plant Triphysaria. Plant Cell 2010, 22(4):1404-1419.

14. Keyes WJ, O'Malley RC, Kim D, Lynn DG: Signaling organogenesis in parasitic angiosperms: xenognosin generation, perception, and response. J Plant Growth Regul 2000, 19(2):217-231.

15. Kim D, Kocz R, Boone L, Keyes WJ, Lynn DG: On becoming a parasite: evaluating the role of wall oxidases in parasitic plant development. Chem Biol 1998, 5(2):103-117.

16. Smith CE, Ruttledge T, Zeng Z, O'Malley RC, Lynn DG: A mechanism for inducing plant development - the genesis of a specific inhibitor. PNAS 1996, 93:6986-6991.

17. Scholes JD, Press MC: Striga infestation of cereal crops - an unsolved problem in resource limited agriculture. Curr Opin Plant Biol 2008, 11(2):180-186.

18. Timko MP, Gowda BS, Ouedraogo J, Ousmane B: In Integrating New Technologies for Striga Control: Towards Ending the Witch-Hunt. Edited by Ejeta G, Gressel J. Singapore: World Scientific; 2007:115-128.

19. Thurmann LD: PhD thesis: Genecological studies in Orthocarpus subgenus Triphysaria (Scrophulariaceae). Berkeley: University of California; 1966

20. Bandaranayake PC, Tomilov A, Tomilova NB, Ngo QA, Wickett N, de Pamphilis CW, Yoder Jl: The TvPirin gene is necessary for haustorium development in the parasitic plant Triphysaria versicolor. Plant Physiol 2012, 158(2):1046-1053.

21. Matvienko M, Torres MJ, Yoder Jl: Transcriptional responses in the hemiparasitic plant Triphysaria versicolor to host plant signals. Plant Physiol 2001, 127(1):272-282.

22. Matvienko M, Wojtowicz A, Wrobel R, Jamison D, Goldwasser Y, Yoder J: Quinone oxidoreductase message levels are differentially regulated in parasitic and non-parasitic plants exposed to allelopathic quinones. Plant J 2001, 25(4):375-387.

23. Li ZH, Wang Q, Ruan X, Pan CD, Jiang DA: Phenolics and plant allelopathy. Molecules 2010, 15(12):8933-8952 
24. Jamison DS, Yoder II: Heritable variation in quinone-induced haustorium development in the parasitic plant Triphysaria. Plant Physiol 2001, 125(4):1870-1879.

25. Palmer AG, Chen MC, Kinger NP, Lynn DG: Parasitic angiosperms, semagenesis and general strategies for plant-plant signaling in the rhizosphere. Pest Manag Sci 2009, 65(5):512-519.

26. Rietveld WJ: Allelopathic effects of juglone on germination and growth of several herbaceous and woody species. J Chem Ecol 1983, 9:295-308.

27. Cao G, Sofic E, Prior RL: Antioxidant and prooxidant behavior of flavonoids: structure-activity relationships. Free Radic Biol Med 1997, 22(5):749-760.

28. Gould KS, Lister C: Flavonoid function in plants. In Flavonoids: Chemistry, biochemistry and applications. Edited by Andersen M, Markham KR. Boca Raton, FL: CRC Press; 2006:397-441.

29. Wang $H$, Cao G, Prior RP: Oxygen radical absorbing capacity of anthocyanins. J Agric Food Chem 1997, 45:304-309.

30. Torres MJ, Tomilov AA, Tomilova N, Reagan RL, Yoder Jl: Pscroph, a parasitic plant EST database enriched for parasite associated transcripts. BMC Plant Biol 2005, 5:24

31. Wickett NJ, Honaas LA, Wafula EK, Das M, Huang K, Wu B, Landherr L, Timko MP, Yoder J, Westwood JH, et al: Transcriptomes of the parasitic plant family Orobanchaceae reveal surprising conservation of chlorophyll synthesis. Current Biology: CB 2011, 21(24):2098-2104.

32. Pang $H$, Bartlam M, Zeng Q, Miyatake H, Hisano T, Miki K, Wong LL, Gao GF, Rao Z: Crystal structure of human pirin: an iron-binding nuclear protein and transcription cofactor. J Biol Chem 2004, 279(2):1491-1498.

33. Murzin AG: Structural classification of proteins: new superfamilies. Curr Opin Struct Biol 1996, 6(3):386-394.

34. Taneja B, Mande SC: Conserved structural features and sequence patterns in the GroES fold family. Protein Eng 1999, 12(10):815-818.

35. Bashton M, Chothia $\mathrm{C}$ : The geometry of domain combination in proteins. J Mol Biol 2002, 315(4):927-939.

36. Rubach JK, Plapp BV: Amino acid residues in the nicotinamide binding site contribute to catalysis by horse liver alcohol dehydrogenase. Biochemistry 2003, 42(10):2907-2915.

37. Thorn JM, Barton JD, Dixon NE, Ollis DL, Edwards KJ: Crystal structure of Escherichia coli QOR quinone oxidoreductase complexed with NADPH. J Mol Biol 1995, 249(4):785-799.

38. Dunning FM, Sun $W$, Jansen $K L$, Helft L, Bent AF: Identification and mutational analysis of Arabidopsis FLS2 leucine-rich repeat domain residues that contribute to flagellin perception. Plant Cell 2007, 19(10):3297-3313

39. Ellis JG, Lawrence GJ, Luck JE, Dodds PN: Identification of regions in alleles of the flax rust resistance gene $L$ that determine differences in gene-for -gene specificity. Plant Cell 1999, 11(3):495-506.

40. McDowell JM, Dhandaydham M, Long TA, Aarts MG, Goff S, Holub EB, Dangl JL: Intragenic recombination and diversifying selection contribute to the evolution of downy mildew resistance at the RPP8 locus of Arabidopsis. Plant Cell 1998, 10(11):1861-1874.

41. Rose LE, Bittner-Eddy PD, Langley $\mathrm{CH}$, Holub EB, Michelmore RW, Beynon JL: The maintenance of extreme amino acid diversity at the disease resistance gene, RPP13, in Arabidopsis thaliana. Genetics 2004, 166(3):1517-1527.

42. Seeholzer S, Tsuchimatsu T, Jordan T, Bieri S, Pajonk S, Yang W, Jahoor A Shimizu KK, Keller B, Schulze-Lefert P: Diversity at the Mla powdery mildew resistance locus from cultivated barley reveals sites of positive selection. Mol Plant Microbe Interact 2010, 23(4):497-509.

43. Yahiaoui N, Brunner S, Keller B: Rapid generation of new powdery mildew resistance genes after wheat domestication. Plant J 2006, 47(1):85-98.

44. Porebski S, Grant Bailey L, Baum BR: Modification of a CTAB DNA extraction protocol for plants containing high polysaccharide and polyphenol components. Plant Mol Biol Report 1997, 15(1):8-15.

45. Livak KJ, Schmittgen TD: Analysis of relative gene expression data using real-time quantitative PCR and the 2(-Delta Delta C(T)) Method. Methods 2001, 25(4):402-408.

46. Altschul SF, Madden TL, Schaffer AA, Zhang J, Zhang Z, Miller W, Lipman DJ: Gapped BLAST and PSI-BLAST: a new generation of protein database search programs. Nucleic Acids Res 1997, 25(17):3389-3402.

47. Corpet F: Multiple sequence alignment with hierarchical clustering. Nucleic Acids Res 1988, 16(22):10881-10890.
48. Larkin MA, Blackshields G, Brown NP, Chenna R, McGettigan PA, McWilliam $H$, Valentin F, Wallace IM, Wilm A, Lopez R, et al: Clustal W and Clustal X version 2.0. Bioinformatics 2007, 23(21):2947-2948.

49. Thompson JD, Higgins DG, Gibson TJ: CLUSTAL W: improving the sensitivity of progressive multiple sequence alignment through sequence weighting, position-specific gap penalties and weight matrix choice. Nucleic Acids Res 1994, 22(22):4673-4680.

50. Tamura K, Peterson D, Peterson N, Stecher G, Nei M, Kumar S: Molecular evolutionary genetics analysis using maximum likelihood, evolutionary distance, and maximum parsimony methods. Mol Biol Evol 2011, 28:2731-2739.

51. Librado P, Rozas J: DnaSP v5: a software for comprehensive analysis of DNA polymorphism data. Bioinformatics 2009, 25:1451-1452.

52. Jukes TH, Cantor CR: Evolution of protein molecules. In Mammalian Protein Metabolism. Edited by Munro HN. New York: Academic; 1969:32-41.

doi:10.1186/1471-2229-13-28

Cite this article as: Ngo et al:: The differentially regulated genes TVQR1 and TvPirin of the parasitic plant Triphysaria exhibit distinctive natural allelic diversity. BMC Plant Biology 2013 13:28.

\section{Submit your next manuscript to BioMed Central and take full advantage of:}

- Convenient online submission

- Thorough peer review

- No space constraints or color figure charges

- Immediate publication on acceptance

- Inclusion in PubMed, CAS, Scopus and Google Scholar

- Research which is freely available for redistribution 